\title{
Aproximación teorética de la concepción educativa de los estudios de postgrado. Un estudio sobre la base de la Teoría Fundamentada
}

\author{
Ivonne Acosta Campos ${ }^{1}$ \\ Elina Marval Galvis ${ }^{2}$ \\ María Elena Pérez Prieto ${ }^{3}$
}

\section{Introducción}

El presente estudio cualitativo tuvo como propósito generar una aproximación teorética de la concepción educativa que sustenta los estudios de Postgrado de la Universidad Nacional Experimental Rafael María Baralt ${ }^{4}$ (UNERMB) a partir de la opinión de sus principales actores, se destaca como marco referencial la concepción de aprendizaje, de investigación y de universidad que tienen los mismos.

El tipo de investigación está enmarcado en una de las diferentes tradiciones cualitativas, específicamente la denominada Teoría

1 Doctora en Ciencias. Mención Gerencia. Magíster en Gerencia de Empresas. Economista. Universidad Nacional Experimental Rafael María Baralt (UNERMB), Venezuela. Adscrita al Centro de Estudios e Investigaciones Sociales, Económicas y Políticas (CEISEP). Línea de Investigación Perspectiva Administrativa, Económica y Gerencial de las organizaciones. Correo: ivonneacostac@hotmail. com.

2 Doctora en Ciencias de la Educación. Magíster en Docencia para Educación Superior. Socióloga. Universidad Nacional Experimental Rafael María Baralt (UNERMB), Venezuela. Línea de Investigación Dimensión Curricular y Gerencial de las Organizaciones. Correo electrónico: elinavictoria@cantv.net.

3 Doctora en Gerencia. Maestría en Gerencia de Recursos Humanos. Licenciada en Administración. Mención Gerencia Industrial. Universidad Nacional Experimental Rafael María Baralt (UNERMB), Venezuela. Coordinadora del CEISEP. Línea de Investigación Perspectiva Administrativa, Económica y Gerencial de las Organizaciones. Correo: perezmariele@hotmail.com.

4

Venezuela 
Fundamentada (TF), creada por los sociólogos Barney Glaser y Anselm Strauss. Para los mencionados autores, esta tradición deriva de datos recopilados de manera sistemática y se analizan por medio de un proceso de investigación que le permite a la teoría emerger a partir de los mismos y no como una teoría preconcebida (Strauss y Corbin, 2002a).

La finalidad de este estudio se concretó en dos aspectos fundamentales:

1. El aporte teórico, se sintetiza en el análisis crítico de posiciones coincidentes y contradictorias, interpretado mediante la compresión de los elementos que sustentan la concepción educativa, de investigación y de universidad, así como también a través de las entrevistas a profundidad, aplicadas a las informantes clave: profesores de la UNERMB, seleccionadas a través de criterios coherentes con la investigación, quienes aportaron información significativa para que emergiera la teoría que permitió generar una aproximación teorética de la concepción educativa que sustenta los estudios de Postgrado de la Universidad Nacional Experimental Rafael María Baralt según la opinión de sus principales actores.

2. Laidentificación de los diferentestemas, propiedadesy matriz condicional que caracterizan la concepción educativa del postgrado de la UNERMB, sobre la base de las perspectivas de las informantes que participaron en el estudio, pues las preguntas de investigación fueron realimentadas durante el proceso investigativo y reformuladas para lograr la saturación de los temas y sus propiedades. Esto permitió a las investigadoras adquirir experticia en el uso de la Teoría Fundamentada, desarrollando un esquema de trabajo para esclarecer las interrogantes iniciales del estudio, debido al poco desarrollo de esta tradición cualitativa en Venezuela. Además de confirmar la viabilidad y pertinencia de la aplicación de la TF como método para abordar el objeto de estudio presentado. 
3. En el Cuadro 1, se presenta un resumen de la aplicación del tema de investigación.

\section{Cuadro 1}

Aplicación al tema de investigación. Aproximación teorética de la concepción educativa de los estudios de postgrado. Un estudio sobre la base de la Teoría Fundamentada

\begin{tabular}{|c|c|}
\hline Categoría & Teoría Fundamentada \\
\hline Foco & $\begin{array}{c}\text { Desarrollar una teoría fundamentada de la } \\
\text { concepción educativa de los estudios de postgrado } \\
\text { de la Universidad Nacional Experimental Rafael } \\
\text { María Baralt. }\end{array}$ \\
\hline $\begin{array}{l}\text { Disciplina de } \\
\text { origen }\end{array}$ & Ciencias Sociales - Ciencias de la Educación. \\
\hline \multirow{2}{*}{$\begin{array}{l}\text { Recolección de } \\
\text { datos }\end{array}$} & $\begin{array}{l}\text { Datos secundarios: a través de la revisión de la } \\
\text { literatura relacionada. } \\
\text { Datos primarios: se utilizó la técnica de la entrevista } \\
\text { a docentes-investigadoras en el área de postgrado y } \\
\text { currículo. }\end{array}$ \\
\hline & $\begin{array}{l}\text { Se utilizó una muestra teórica (intencional) de } 5 \\
\text { informantes clave, para obtener información sobre } \\
\text { las categorías necesarias para generar la teoría: } \\
\text { fenómeno central, condiciones causales, estrategias, } \\
\text { contexto y consecuencias. }\end{array}$ \\
\hline Análisis de datos & $\begin{array}{c}\text { Se establecieron y generaron las codificaciones: } \\
\text { abierta - axial - selectiva }\end{array}$ \\
\hline Forma narrativa & $\begin{array}{l}\text { Representación gráfica y teórica de la concepción de } \\
\text { los estudios de Postgrado de la UNERMB }\end{array}$ \\
\hline
\end{tabular}

\section{Desarrollo del estudio}

Para emprender la indagación y dirigir el muestreo teórico, se realizó una pregunta principal de investigación con la finalidad de profundizar el entendimiento de los contenidos teóricos. Las preguntas secundarias, según la tradición de la TF fueron utilizadas para construir el guion de entrevistas y hacer comparaciones a través de las diferentes codificaciones, de acuerdo a las opiniones de las informantes clave (Strauss y Corbin, 2002b). 
Pregunta principal:

¿Cuál es la concepción educativa que fundamenta los estudios de postgrado de la UNERMB?

Preguntas secundarias:

¿Cuáles cree usted son las teorías de aprendizajes que sustentan la concepción educativa de los estudios de postgrado de la UNERMB?

¿Cuál concepción tienes de investigación?

¿Cuál concepción tienes de universidad?

Las preguntas de investigación parten de la asunción que tienen las investigadoras sobre el fenómeno de estudio, en primer término, por los comentarios obtenidos a través de entrevistas con los informantes clave (docentes de la UNERMB) y, en segundo lugar, mediante las teorías expuestas por diferentes autores. En este estudio la asunción es la siguiente: la concepción educativa que sustenta los estudios de postgrado de la UNERMB, orientan el aprendizaje de los actores del hecho educativo, de universidad y de investigación, que exige la transformación social y académica para la construcción de un individuo integral.

\section{Asunción de la investigación}

Mediante el estudio de cómo se deben abordar los procesos de aprendizajes en los postgrados, así como la concepción que tiene de investigación y de universidad sus principales actores, puede emerger una teoría fundamentada acerca de la concepción educativa de los estudios de postgrado de la Universidad Nacional experimental Rafael María Baralt.

\section{Descripción de la Teoría Fundamentada y su aplicación al objeto de estudio}

El carácter cualitativo de esta tradición investigativa tiene como plataforma la generación de teoría sobre la realidad estudiada, a partir del proceso de teorización, el investigador descubre categorías abstractas y relaciones entre éstas, para desarrollar o confirmar las explicaciones del cómo y el por qué de los fenómenos. Esto permite 
formular una teoría que se encuentra subyacente en los datos obtenidos de la realidad investigada.

Antes de explicar la plataforma que soporta la TF, es importante referir que esta tiene sus raíces epistemológicas en el interaccionismo simbólico o Escuela de Chicago, pues sus fundadores, Barney Glaser y Anselm Strauss, estuvieron influenciados e inspirados de los escritos interaccionistas y pragmatistas de Dewey (1922), Mead (1934), Thomas (1966), Park (1967), Blume (1969) y Hughes (1971), tal como lo manifiestan Strauss y Corbin (2002b, p. 10). A pesar de la diversidad de aspectos existentes en este enfoque metodológico existen tres postulados comunes que hacen del interaccionalismo una concepción sólida (Woods, 1979, citado por Rusque, 1999, p. 68):

1. Los seres humanos actúan sobre las cosas en función del sentido que le atribuyen. En consecuencia, los interaccionistas centran sus reflexiones en el mundo de los significados subjetivos y en los símbolos por los cuales los significados se producen.

2. Esa atribución de significados a los objetos a través de símbolos es un proceso continuo que se produce como un flujo y está sujeto a cambios. La acción es determinada tanto por atributos psicológicos como por factores sociales externos.

3. Ese proceso se realiza en un contexto social. Plantea el autor que cada individuo dirige su acción hacia otros, se coloca en su lugar, intenta dirigir las impresiones que los otros tienen de él, puede tratar de influir en ellos y de asumir un rol ante la definición de determinadas situaciones.

Es importante exponer algunas consideraciones que se desprenden de estos postulados. La vida es un continuo movimiento. Las personas, de acuerdo al momento histórico, producen sus propias acciones y significados, exponiendo sus creencias ante las diversas situaciones que viven, las comparten y definen su cosmovisión al respecto. Esta experiencia convivencial conlleva a un aprendizaje que define su comportamiento manifestado en símbolos permitiendo su interacción con otros. Para Mead (1932, citado por Rusque, 1999, 
p. 68), un símbolo es "un estímulo que tiene un sentido y un valor aprendido por las personas que reaccionan en función de ese sentido y su valor". Así van construyendo la situación en que se encuentran, modificándola, articulándola de acuerdo al devenir de los momentos históricos.

Ahora bien, la necesidad de seleccionar el método cualitativo para este estudio y la tradición de la TF, se cimentó en los siguientes argumentos:

1. La característica definitoria de esa tradición es que las proposiciones teóricas no se postulan al inicio del estudio, las generalizaciones emergen de los propios datos y no se forman previa la recolección de los mismos (Mertens, 1998, citado en Sandín, 2003). Si bien es cierto, existe un abordaje sobre la concepción de los estudios de postgrado, no se ha conseguido una representación teórica de la concepción educativa que los sustente. La teoría que emerja puede asumir la forma de una narración, una figura visual o una serie de hipótesis o proposiciones (Creswell, 1998).

2. La base de la TF es la "creencia que el significado del fenómeno de estudio se deriva de la interacción entre los individuos y el contexto" (Creswell, citado en Vera, 2003a, p. 10). Entonces, podemos inferir que esta teoría permite la interacción de las informantes clave y detecta de esa manera sus posturas, acciones y cómo reaccionan ante ese fenómeno.

3. Para Strauss y Corbin(2002a, p. 13), la Teoría Fundamentada es una método general para desarrollar teoría, a través de una continua interpelación entre el análisis sistemático y la recogida de los datos, usando un acercamiento orientado hacia el constructo o categoría, donde es conveniente que el investigador deje de lado sus ideas teóricas o nociones para que la teoría analítica pueda emerger.

Es importante señalar que Glaser y Strauss (1967) distinguen entre teoría sustantivas referida a la desarrollada para un área empírica como, por ejemplo, manejo de emociones, procesos de aprendizaje; 
y la teoría formal que se desarrolla para un área conceptual de la indagación social como la socialización, la movilidad social. Estos autores defendían la utilidad de las teorías sustantivas o de medio rango para explicar un área específica de conocimiento puesto que las teorías formales utilizan conceptos globales que resultan no ser aplicables fácilmente para dar sentido y explicar cuestiones prácticas de la investigación social.

De allí, la importancia de este método inductivo de investigación cualitativa puesto que ofrece un enfoque sistemático de generación de teorías sustantivas que se construyen para explicar un fenómeno de estudio específico. Asume Sandín (2003, p. 153) que la TF "va más allá de las teorías y marcos conceptuales preconcebidos existentes, en búsqueda de nuevas comprensiones de los procesos sociales desarrollados en contextos naturales".

En la metodología cualitativa de la Teoría Fundamentada, el momento analítico se compone de cuatro (4) procesos; los mismos pueden ser usados de manera mecánica y en cualquier sentido, así como omitirse o modificarse de ser necesario, es decir, no están sujetos al cuándo, dónde y cómo, no obstante, según Strauss \& Corbin (2002a) y Vera (2003a), se pueden señalar de la siguiente manera:

1. Codificación abierta: proceso analítico a través del cual se identifican los conceptos y se descubren en los datos sus propiedades y dimensiones. En este momento, los datos se descomponen en partes discretas y se identifican las categorías más notables, saturándolas hasta no obtener más información de las mismas. Este procedimiento permite reducir la data a un conjunto de temas manejables, o categorías más abstractas, para darle paso al segundo proceso analítico de la data.

Las categorías son "conceptos derivados de los datos, que representan los fenómenos" (Strauss \& Corbin, 2002a, p. 124). Los nombres de las categorías provienen del conjunto de los conceptos que se descubren en los datos. Cuando el investigador examina la lista de los conceptos, es probable que algunos se destaquen por ser más amplios y abstractos que los demás. Señalan los autores, que es 
significativo que una vez que los conceptos comienzan a acumularse, el analista o investigador debe iniciar el proceso de agruparlos sobre la base de términos explicativos más abstractos, o sea, en categorías. El proceso del análisis de las categorías conlleva a familiarizarse con ella para definirla, facilitando su comprensión para desarrollarla, es decir, descomponiéndola en términos de sus propiedades explicando los cuándo, dónde, por qué y cómo pudieran existir en una categoría.

2. Codificación axial: proceso de relacionar las categorías a subcategorías, ocurre alrededor del eje de una categoría enlazándolas entre sí según sus propiedades y dimensiones. En este momento se reagrupan los datos obtenidos en la codificación abierta, y se integran de un solo conjunto de información significativa para la totalidad de los datos, en un nivel aún más abstracto.

3. Codificación selectiva: proceso de integrar y refinar la teoría. En este momento las categorías principales se relacionan y dan sentido de conjunto permitiendo que emerja la teoría, para determinar el punto central de la misma.

4. Matriz condicional o consecuencial: es un mecanismo analítico que estimula el pensamiento del investigador sobre las relaciones entre las condiciones, y las consecuencias micro: limitadas en su alcance y posible impacto, o macro: aquellas amplias en su alcance potencial, tanto entre ellas como para el proceso. En este momento se recurre a las sendas de conectividad como la forma compleja en donde las condiciones y consecuencias micro y macro se entrecruzan para crear un contexto sobre la acción e interacción que especifica la naturaleza general de los acontecimientos y fenómenos significativos.

Esos momentos constituyen el procedimiento de análisis de los datos de esta investigación en su contexto real, para poder generar la teoría y extraer de ellos los significados esclareciéndolos a través de la matriz condicionada.

Por otra parte, Glasser y Strauss (1967) proponen dos (2) estrategias fundamentales para que emerja la teoría sustantiva: el método de la comparación constante y el muestreo teórico. A través del método de la comparación constante el investigador codifica y 
analiza los datos de forma simultánea para desarrollar conceptos. Su aplicación supone una contrastación de las categorías, propiedades e hipótesis que surgen a lo largo de un estudio en sucesivos contextos. Este procedimiento se desarrolla en cuatro (4) etapas:

1. La primera implica la comparación de los datos,

2. La segunda supone una integración de cada categoría con sus propiedades;

3. La tercera requiere delimitar la teoría que comienza a desarrollarse, y por último,

4. La cuarta etapa recoge la redacción de la teoría.

Finalmente, el otro criterio, también de mucha importancia, es la independencia, cuyo propósito es examinar si los enunciados teóricos y los datos empíricos recogidos en el espacio informacional se superponen unos a otros, o si alguno de ellos está implícito o subyacente en otros. En esta situación, se estarían confundiendo los niveles de razonamiento y estructuración del modelo diseñado con respecto a los hechos concretos.

\section{Validez de la investigación}

En cuanto a los criterios de validez, se utilizaron los descritos por Maxwell (1996), tal como se especifica a continuación:

La validez descriptiva: se realizó para validar la descripción de la data en forma precisa y concreta, utilizando como técnica la grabación de las entrevistas y posteriormente su trascripción.

La validez interpretativa, se hizo a través de los registros (memos) para evitar la imposición de prejuicios y valores del mismo sobre la data y la validez teorética, permitió revelar las contradicciones para ofrecer explicaciones alternativas y comprender el evento, se analizaron las divergencias que emergieron de la data y se redactaron esclarecimientos alternativos para la comprensión del mismo. 


\section{Técnicas y procedimiento para la recolección de la data}

La recolección de la data en esta investigación estuvo orientada por los criterios que sustentan la TF, es decir, en función del proceso denominado muestreo teórico, tal cual, lo definen Strauss y Corbin (2002a, p. 219):

Recolección de datos guiada por los conceptos derivados de la teoría que se está construyendo y basada en el concepto de hacer comparaciones, cuyo propósito es acudir a lugares, personas o acontecimientos que maximicen las oportunidades de descubrir variaciones entre los conceptos y que hagan más densas las categorías en términos de sus propiedades y dimensiones.

Este proceso tiene lugar en las fases iniciales de la investigación. A lo que respecta, se recogió, codificó y analizó la data que se iba obteniendo, decidiendo qué datos escoger y dónde encontrarlos, a fin de desarrollar la teoría tal como iba emergiendo. Previo a esto se establecieron los criterios para la selección de los informantes clave que proporcionen información pertinente, cuándo visitarlos, así como las fuentes de información necesarias para la revisión de documentación relacionada con la investigación.

Es importante indicar que este proceso de recolección está en función de la teoría emergente sea esta sustantiva o formal. El muestreo teórico es acumulativo. Cada acontecimiento que entra en el muestreo se incorpora al análisis hasta que se sature la información. Es un ir y venir, es establecer comparaciones constante y regresar a las fuentes tantas veces sea necesario para construir la teoría, es decir el muestreo y el análisis deben ocurrir de manera secuencial y el análisis es el que indica la selección de los datos. Al inicio del proceso se generaron un gran número de posibles categorías, las cuales fueron desarrolladas, haciéndolas más densas hasta saturarlas.

Al igual que otros métodos cualitativos en la Teoría Fundamentada, las técnicas de recolección de datos son: las observaciones de campo y la entrevista, así como material impreso de todo tipo y las grabaciones audiovisuales. En este estudio prevalece la entrevista a profundidad a través de la cual se pudo dialogar 
directamente con las informantes clave, expertos en materia de currículo, investigación y postgrado, descubrir sus concepciones, pensamientos, supuestos y creencias.

Las preguntas de la entrevista inicial se basaron en conceptos derivados de la literatura, de la experiencia de las investigadoras y de las observaciones de campo, las cuáles fueron cambiando en la medida en que fueron emergiendo los nuevos datos, permitiendo de esa manera darle flexibilidad a la guía de entrevista para conseguir el tipo y la cantidad de datos necesarios para generar la teoría. En este tipo de investigación es necesario utilizar instrumentos no estructurados, pues de lo contrario los entrevistados se limitarían a responder solo lo que se les solicita y a veces ni los responden completamente.

Con relación a los informantes clave, éstos pasan a ser fuentes primarias de información al complementar los conocimientos sobre el evento seleccionado, identificados previamente a través de los criterios de selección como las personas ideales, afines a la investigación (Taylor y Bogan, 1990). Se contó con cinco (5) docentes de la UNERMB, los cuales manifestaron su agrado en participar en la investigación, ninguno planteó mantenerse en el anonimato, sin embargo, consideramos pertinente no mencionar sus nombres. A continuación se hace una breve descripción de su data demográfica.

Dos (2) son docentes adscritas a los programas de pregrado con desempeño en el postgrado y tres (3) a dedicación exclusiva de postgrado. La edad oscila entre 50 y 55 años. Todos son investigadores activos del postgrado, cuatro son especialistas en currículo. Los criterios de selección de las informantes fueron guiados por la pregunta principal de la investigación: experiencia por más de cinco (5) años en postgrado (investigación y/o currículo).

Procedimientos para el análisis de la data

El procedimiento para el análisis de la data resultó interesante, pero no surge en un instante, los datos no emergen solos, es un proceso sistemático; se va logrando con el tiempo y continuidad, a través de leer y releer la data, es una interacción directa entre el investigador y los datos, así como la evolución del pensamiento, el cual ocurre en la 
medida que emergen los datos y se acumula el cuerpo de hallazgos (Marval y Valera, 2007). Es importante señalar que solo se presentan extractos de los datos suministrados por las informantes clave, debido a que este artículo constituye un resumen de la investigación. A continuación se describe paso a paso las técnicas utilizadas para analizar la data.

Primer paso. Fase de codificación abierta: se procedió a codificar la data obtenida en las trascripciones de las entrevistas, se seleccionaron los elementos significativos e identificaron las categorías o temas, así como las propiedades. En la codificación abierta se muestra el extracto de las ideas centrales de los datos, y se interpretan como conceptos solo los extractos. Strauss \& Corbin (2002a), opinan que los mismos son la base fundamental para desarrollar teoría. A través de la fase de comparación constante se redujo la data a un conjunto de temas y propiedades manejables, a fin de saturarlas hasta no obtener más información.

De la data recopilada emergieron en la codificación abierta (7) temas o categorías: (a) procesos andragógicos, (b) teoría humanista, (c) teoría constructivista, (d) teoría crítica, (e) educación en la diversidad, (f) educación interdisciplinaria y transdisciplinaria y (g) red y relaciones interconectada con la comunidad y el entorno, tal como se observa en el Cuadro 2.

Segundo paso. Fase de codificación axial: permitió visualizar las relaciones entre las propiedades de los temas para integrarlos en categorías más abstractas. La representación la realizamos a través de la técnica del flechado, con la finalidad de mostrar la relación de ideas y su reciprocidad. Como se observa, surgieron cuatro (4) temas: (a) teorías del aprendizaje, (b) pertinencia académica, (c) abordaje del conocimiento, y (d) pertinencia social. Estos temas se derivan inductivamente como producto de la teoría existente, de la creatividad y experiencia de las investigadoras, de manera que reflejen con mayor precisión los conceptos que éstas representan. En el Gráfico 1, se muestra una representación visual de temas y propiedades que emergieron de la data y en el Cuadro 3, se refleja la codificación axial. 


\section{Cuadro 2}

Codificación abierta. Concepción educativa que fundamenta los estudios de postgrado (solo se presentan extractos) según la opinión de sus principales actores

\begin{tabular}{|c|c|c|c|}
\hline $\mathrm{N}^{\circ}$ & DATA & $\begin{array}{c}\text { TEMAS O } \\
\text { CATEGORÍAS }\end{array}$ & PROPIEDADES \\
\hline 1 & $\begin{array}{l}\text { "Es necesario implementar los } \\
\text { procesos andragógicos, donde } \\
\text { se incentiven la autorreflexión, } \\
\text { la autodeterminación y la } \\
\text { responsabilidad en los participantes" } \\
\text { "Hay autores que muy bien } \\
\text { plantean... como debe ser la } \\
\text { educación de postgrado, porque } \\
\text { inclusive, si nos vamos hasta } \\
\text { la misma escuela básica se está } \\
\text { hablando... de que el niño sea dueño } \\
\text { de su propio aprendizaje,..., entonces } \\
\text { en el postgrado hay que trabajar } \\
\text { con ese tipo de estrategia pues en } \\
\text { el postgrado, específicamente en } \\
\text { la maestría y el doctorado se debe } \\
\text { formar investigadores". } \\
\text { "La concepción educativa para el } \\
\text { postgrado debe estar sustentada en } \\
\text { una educación de adultos, en los } \\
\text { procesos andragógicos... } \\
\text { "Concretar la educación continua en } \\
\text { los procesos andragógicos y... todo } \\
\text { lo que es el concepto de adulto como } \\
\text { tal". } \\
\text { "El postgrado está sustentado en } \\
\text { el Modelo de Hecho Educativo, } \\
\text { que dentro de sus concepciones } \\
\text { filosóficas o sus concepciones está } \\
\text { enmarcado dentro de las ideas de la } \\
\text { andragogía, la educación de adultos, } \\
\text { la educación continua, donde la } \\
\text { autorrealización, la responsabilidad } \\
\text { del adulto, debe ser el quehacer } \\
\text { del individuo en su proceso de } \\
\text { aprendizaje, él es el responsable de su } \\
\text { propio aprendizaje". }\end{array}$ & $\begin{array}{c}\text { Procesos } \\
\text { andragógicos }\end{array}$ & $\begin{array}{c}\text { Autorreflexión, } \\
\text { Autodeterminación } \\
\text { Responsabilidad } \\
\text { Autorrealización } \\
\text { Dueño de su } \\
\text { propio aprendizaje } \\
\text { Estrategias no } \\
\text { tradicionales } \\
\text { Formación de } \\
\text { investigadores } \\
\text { Manejo de ciertas } \\
\text { estrategias }\end{array}$ \\
\hline
\end{tabular}


Aproximación teorética de la concepción educativa de los estudios de postgrado.

Un estudio sobre la base de la Teoría Fundamentada

\begin{tabular}{|c|c|c|c|}
\hline $\mathbf{N}^{\circ}$ & DATA & $\begin{array}{c}\text { TEMAS O } \\
\text { CATEGORÍAS }\end{array}$ & PROPIEDADES \\
\hline 2 & $\begin{array}{l}\text { "la teoría humanista porque habla } \\
\text { de centrarse en el individuo, la } \\
\text { educación como posibilidad de } \\
\text { autorrealización del ser humano" }\end{array}$ & $\begin{array}{c}\text { Teoría } \\
\text { humanista }\end{array}$ & $\begin{array}{l}\text { 1. Centrarse en el } \\
\text { individuo } \\
\text { 2. Autorrealización } \\
\text { del ser humano }\end{array}$ \\
\hline 3 & $\begin{array}{l}\text { "Por el otro lado en uno de los } \\
\text { documentos se dio como nuevos } \\
\text { aportes, la idea de incorporar } \\
\text { elementos como el Constructivismo, } \\
\text { el conocimiento como proceso de } \\
\text { construcción individual y colectiva, } \\
\text { eso también me parece parte } \\
\text { importante de ese nuevo modelo". } \\
\text { "Me inclino por el constructivismo, } \\
\text { pero sin caer en ortodoxia, porque } \\
\text { ello se contrapone a la esencia misma } \\
\text { de la Universidad, como reservorio } \\
\text { de pluralidad de saberes". } \\
\text { "Aparte de ello, algo muy } \\
\text { importante del modelo que } \\
\text { identifica el Postgrado UNERMB } \\
\text { son las estrategias de aprendizajes, } \\
\text { sustentadas en lo que es el } \\
\text { constructivismo en lo psicosocial, lo } \\
\text { transpersonal sería el único aditivo } \\
\text { que le faltaría a ese modelo, la parte } \\
\text { espiritual y en algún momento } \\
\text { debe ser tomado, pero ya algunos } \\
\text { profesores lo estamos considerando". }\end{array}$ & $\begin{array}{c}\text { Teoría } \\
\text { constructivista }\end{array}$ & $\begin{array}{l}\text { 1. Proceso de } \\
\text { construcción } \\
\text { individual y } \\
\text { colectiva } \\
\text { 2. Psicosocial } \\
\text { 3. Transpersonal } \\
\text { 4. Espiritual }\end{array}$ \\
\hline
\end{tabular}




\begin{tabular}{|c|c|c|c|}
\hline $\mathrm{N}^{\circ}$ & DATA & $\begin{array}{c}\text { TEMAS O } \\
\text { CATEGORÍAS }\end{array}$ & PROPIEDADES \\
\hline 4 & $\begin{array}{l}\text { "También asumir la teoría crítica de } \\
\text { la educación ya que tiene elementos } \\
\text { fundamentales como por ejemplo } \\
\text { el papel del docente como un } \\
\text { intelectual reflexivo, la importancia } \\
\text { que le da a la investigación que } \\
\text { haga el propio docente sobre lo que } \\
\text { ocurre con su práctica cotidiana, esos } \\
\text { son aportes importantísimos que la } \\
\text { teoría crítica de la educación da". } \\
\text { "Yo como soy fiel seguidora de las } \\
\text { teorías críticas de la educación allí se } \\
\text { hace mucho énfasis, no tanto en la } \\
\text { investigación como proyecto de algo, } \\
\text { sino incluso la investigación que uno } \\
\text { debe hacer, la que uno mismo hace. } \\
\text { Esa investigación de la práctica de la } \\
\text { cotidianidad del aula es fundamental } \\
\text { y yo trato de hacerla en la medida de } \\
\text { lo posible, es decir, yo me planteo } \\
\text { que cada curso que uno tiene es una } \\
\text { posibilidad de investigar... }\end{array}$ & Teoría crítica & $\begin{array}{l}\text { l. Docente como } \\
\text { un intelectual } \\
\text { reflexivo. } \\
\text { 2. Docente como } \\
\text { investigador en su } \\
\text { práctica cotidiana }\end{array}$ \\
\hline 5 & $\begin{array}{l}\text { "También asumir los principios que } \\
\text { señala la UNESCO, la educación en } \\
\text { la diversidad, se ha visto la manera } \\
\text { tradicional, debemos unificar, ver las } \\
\text { cosas de manera homogénea. Ahora } \\
\text { sería el respeto a la diversidad". }\end{array}$ & $\begin{array}{c}\text { Educación en la } \\
\text { diversidad }\end{array}$ & $\begin{array}{l}\text { 1. Respeto a la } \\
\text { diversidad }\end{array}$ \\
\hline 6 & $\begin{array}{l}\text { "Debe ser una educación inter y } \\
\text { transdisciplinaria, hace mucho } \\
\text { tiempo uno se ubicaba en un } \\
\text { postgrado enmarcado estrictamente } \\
\text { dentro de una disciplina, ahora uno } \\
\text { ve la posibilidad de pensar en un } \\
\text { postgrado ya sea especialización, } \\
\text { maestría o doctorado bajo otra óptica } \\
\text { que sea mucho más amplia y que no } \\
\text { pierda ese nivel de profundidad y de } \\
\text { especialización..." }\end{array}$ & $\begin{array}{c}\text { Educación } \\
\text { interdisciplinaria } \\
\text { y } \\
\text { transdisciplinaria }\end{array}$ & $\begin{array}{l}\text { 1. Óptica más } \\
\text { amplia }\end{array}$ \\
\hline
\end{tabular}




\begin{tabular}{|c|c|c|c|}
\hline $\mathrm{N}^{\circ}$ & DATA & $\begin{array}{c}\text { TEMAS O } \\
\text { CATEGORÍAS }\end{array}$ & PROPIEDADES \\
\hline 7 & $\begin{array}{l}\text { "La Universidad debe tener un } \\
\text { espacio amplio, un espacio donde } \\
\text { la reflexión, la investigación, } \\
\text { la docencia, la extensión y la } \\
\text { productividad debe ser, tiene que } \\
\text { ser el deber ser y donde exista } \\
\text { realmente la coherencia entre las } \\
\text { expectativas de una sociedad y lo } \\
\text { que la universidad le ofrece a esa } \\
\text { sociedad para poder atender sus } \\
\text { necesidades... lo que demanda } \\
\text { nuestra Constitución Nacional, que } \\
\text { es formar a un individuo crítico, } \\
\text { pensante, socializado, con un } \\
\text { entendimiento de lo que debe ser la } \\
\text { sensibilidad social y responder a esas } \\
\text { expectativas y a esas necesidades. } \\
\text { "Red de relaciones interconectadas } \\
\text { con la comunidad, con el entorno } \\
\text { y ahora más con el asunto de la } \\
\text { globalización, o sea, todo lo que tiene } \\
\text { que ver con la coherencia interna } \\
\text { institucional como con su entorno, } \\
\text { eso es básico, entonces tienen que } \\
\text { haber, me imagino, mecanismos, } \\
\text { puentes como sea de enlace porque } \\
\text { un postgrado desarticulado del } \\
\text { pregrado para mí no tiene sentido... }\end{array}$ & $\begin{array}{c}\text { Red de } \\
\text { relaciones } \\
\text { interconectadas } \\
\text { con la } \\
\text { comunidad y el } \\
\text { entorno }\end{array}$ & $\begin{array}{l}\text { Datisfacer las } \\
\text { expectativas de una } \\
\text { sociedad } \\
\text { 2. Satisfacer las } \\
\text { necesidades de } \\
\text { aprendizaje y } \\
\text { conocimiento. }\end{array}$ \\
\hline
\end{tabular}

Tercer paso: Fase de codificación selectiva: contiene las condiciones relevantes que surgen de la investigación y constituye el tercer paso del momento analítico, identificando las líneas maestras de la concepción educativa expresada por las informantes, y se procedió, como lo plantea Vera (2003b, p. 158), "a efectuar una descripción narrativa que integran las categorías narradas en un modelo de codificación axial". La línea más representativa fue el marco teórico conceptual como plataforma de sustento de los estudios de postgrado de la UNERMB. A partir de este paso se construye la matriz condicional. 


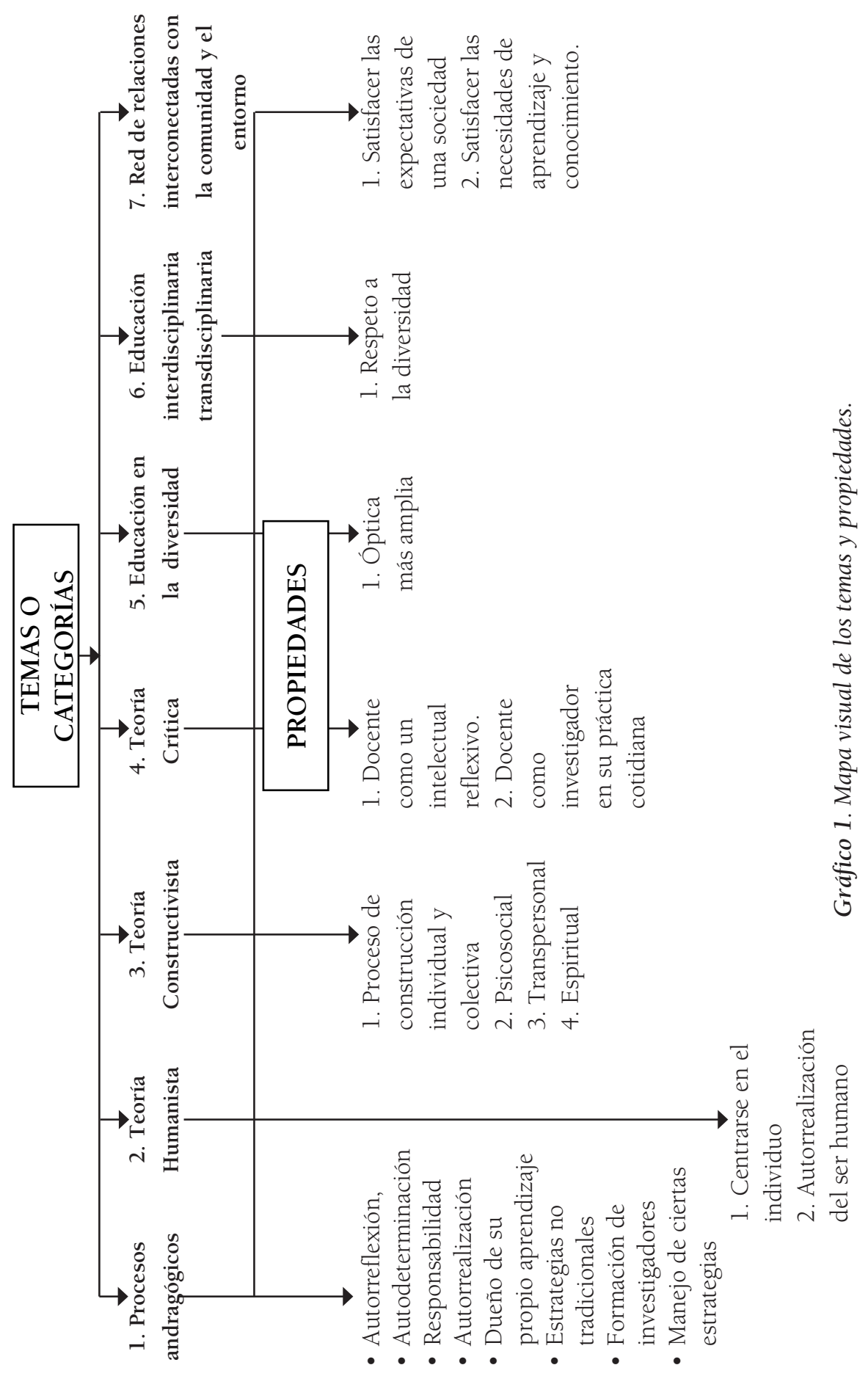


Aproximación teorética de la concepción educativa de los estudios de postgrado. Un estudio sobre la base de la Teoría Fundamentada

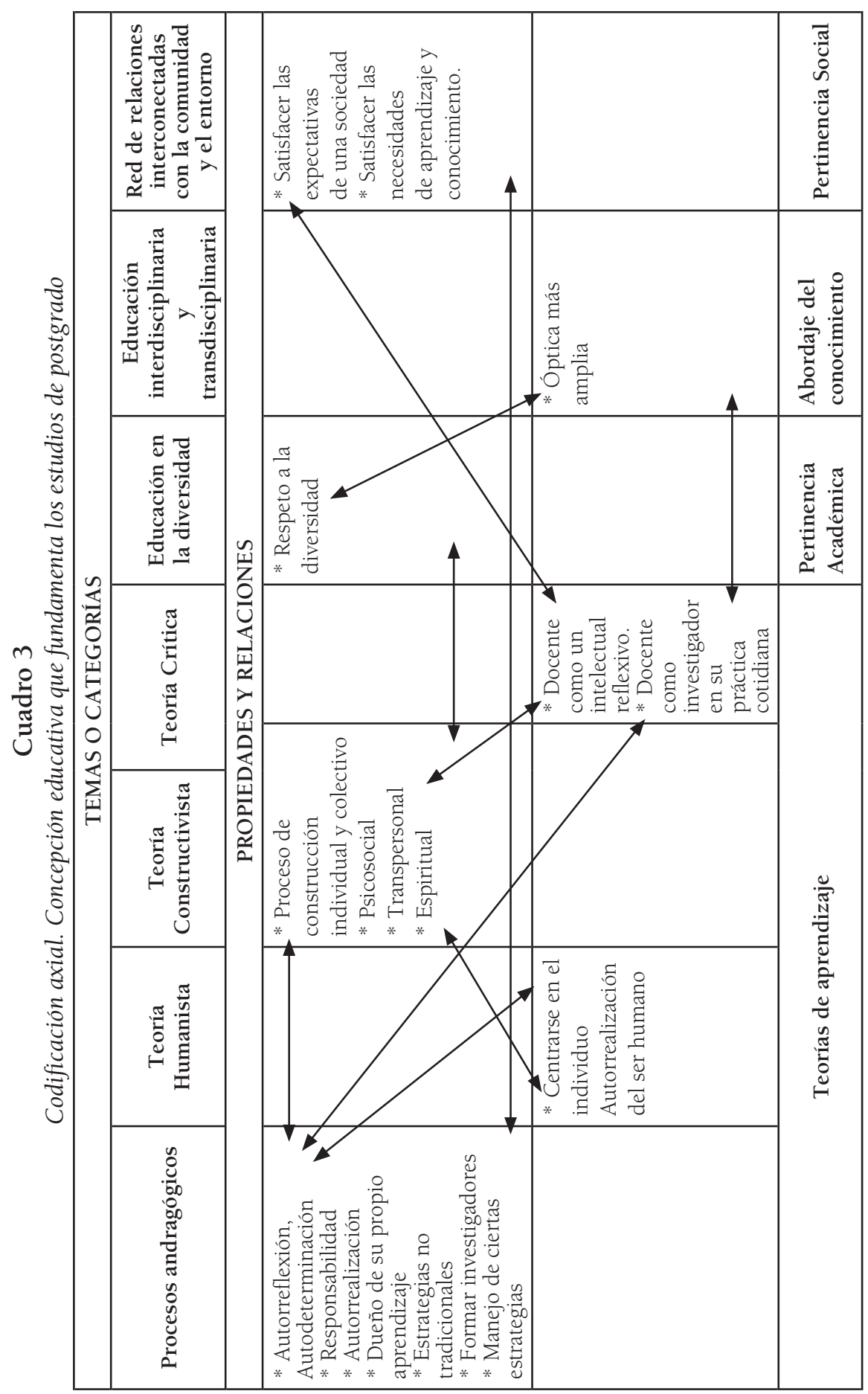


Cuarto paso: matriz condicional: constituye una representación gráfica de los datos reintegrados y de los cuales emergen las conceptualizaciones teoréticas que explican el objeto de estudio. En el Gráfico 2, se muestra con un ejemplo, las condiciones que soportan la concepción educativa de los estudios de postgrado de la UNERMB, según la perspectiva de las informantes clave.

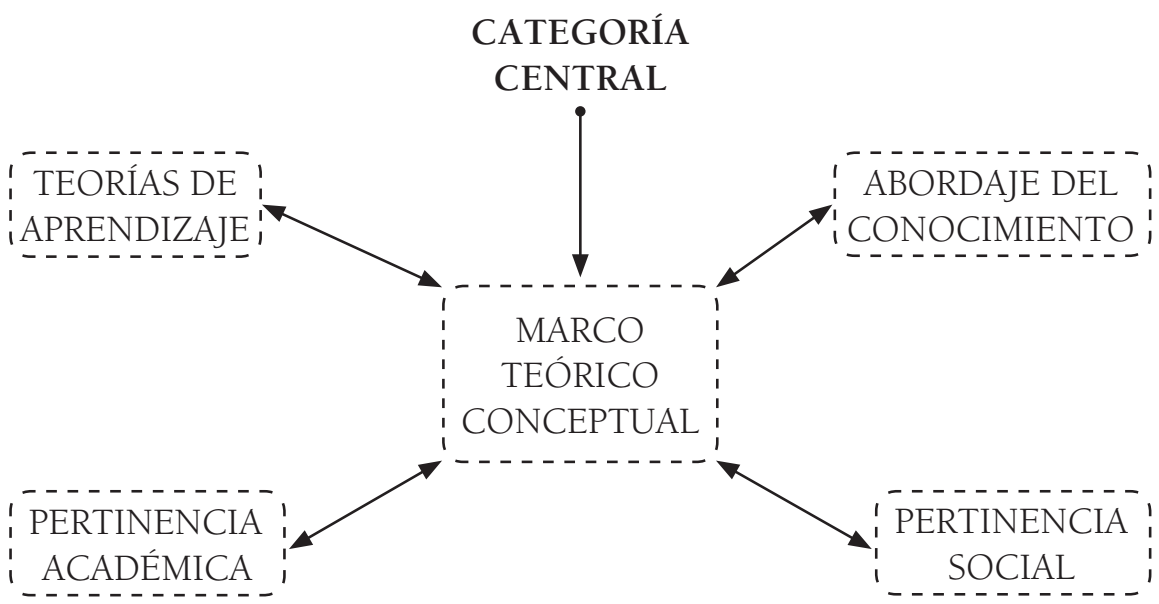

EL MARCO TEÓRICO CONCEPTUAL ES LA PLATAFORMA EN LA CUAL SE FUNDAMENTA LOS ESTUDIOS DE POSTGRADO DE LA UNERMB

Gráfico 2. Matriz condicional derivada de la codificación selectiva

\section{Hallazgos e interpretaciones teóricas}

Del análisis de la data se identificaron once (11) condiciones presente en el fenómeno de estudio, que influyen directamente en los temas principales y las propiedades extraídas de las entrevistas aplicadas a las informantes clave. Existen unas condiciones previas o una manera conceptual de agrupar las respuestas a las siguientes preguntas: por qué, dónde, cuándo y cómo. Los hallazgos emergen de esas condiciones y se discuten de manera separada, los cuales se mencionan a continuación:

Hallazgo 1: los informantes clave comparten la creencia que los estudios de postgrado deben sustentarse en procesos andragógicos, en una educación de adultos, consideran que las personas que ingresan a un postgrado tienen una preparación previa, traen una 
carrera específica y hasta cierto punto tienen una madurez intelectual. Manifestaron: "el postgrado está sustentado en el Modelo de Hecho Educativo Integrativo (MHEI), el cual tiene entre sus concepciones filosóficas las ideas de la andragogía, la educación de adultos y la educación continua".

Hallazgo 2: según los informantes, en los estudios de postgrado se debe incentivar el desarrollo de valores para que el individuo pueda emprender su propio proceso de aprendizaje, donde se incentive la autorreflexión, la autodeterminación y la responsabilidad en los participantes. Sus aportes coinciden con lo planteado por Marval y Acosta (2001), el propósito de los postgrados debe ser generar conocimiento y propiciar en los postgraduados la reflexión crítica y una comprensión amplia del saber, que les permita resolver problemas complejos e interdisciplinarios, indagar el por qué de las cosas, dirigir y trabajar en equipo, comprender nuevos lenguajes y vincularse conscientemente a la problemática local, nacional e internacional.

Hallazgo 3: de igual manera, asumen que los estudios de postgrado deben reafirmar lo aprendido desde la educación preescolar y la educación básica, es decir, la apropiación del aprendizaje por parte del individuo, a través de la aplicación de estrategias no convencionales. El desarrollo de ese proceso permite, desde su perspectiva, el logro de uno de los objetivos de este nivel educativo como es la formación de investigadores.

Hallazgo 4: comparten la creencia que en los estudios de postgrado se debe enfatizar en los principios de la teoría humanista, centrarse en el individuo, y ver la educación como posibilidad de autorrealización del ser humano. Estas reflexiones coinciden con lo planteado por Román y Peña (2006), el paradigma humanista considera a los alumnos como entes individuales, únicos y diferentes de los demás. Son seres con iniciativa, con necesidades personales de crecer, con potencialidad para desarrollar actividades y solucionar problemas creativamente.

Hallazgo 5: consideran, además, la teoría constructivista, por considerar que el conocimiento es producto de una acción individual y colectiva, y a la universidad como reservorio de pluralidad de 
saberes. Aparte de ello, expresaron algo muy importante del Modelo de Hecho Educativo Integrativo (MHEI) del Postgrado de la UNERMB como son las estrategias de aprendizajes, pues está sustentado en el constructivismo, en lo psicosocial, y el único aditivo que le falta al MHEI es el elemento transpersonal, la parte espiritual, aunque algunos profesores lo están considerando, creencia válida si pensamos que la educación busca mejorar cada vez más las condiciones de vida de los individuos. Sus ideas coinciden con los aportes de Román y Peña (2006), para quienes el constructivismo de hoy enfatiza de manera particular "el aprender a aprender" sobre contenidos específicos, tomando conciencia, no solo del propio proceso, sino también, de los valores implicados y las repercusiones de las acciones personales en el contexto.

Hallazgo 6: la mayoría de los informantes coinciden al expresar que la teoría crítica debe sustentar los estudios de postgrado, porque aporta elementos importantes como es el hecho de concebir al docente como un intelectual reflexivo y un investigador activo. Ésta le da mucha importancia a la investigación y a la práctica cotidiana del docente. Al respecto, Morles (2007) plantea el importante papel que el postgrado debe cumplir dentro de las políticas de desarrollo científico y tecnológico, debido a los fuertes cuestionamientos hacia éstos, por su concepción tecnoeconomicista y cientificista, por sus graduados con especialización estrecha, la proletarización de los intelectuales y su función reproductora de la estratificación social imperante.

Hallazgo 7: conciben a la investigación como el eje nuclear de los estudios de postgrado de cualquier universidad, por ser el nivel para formar investigadores al servicio de la sociedad. Comparten la creencia de que la investigación debe hacerse desde el aula; y el docente debe verse como un intelectual reflexivo y como investigador en su práctica cotidiana. Manifiesta una informante que el docente debe ser investigador para poder orientar el proceso investigativo y mejorar su praxis diaria; en cada curso tiene la posibilidad de investigar, de sacar cuáles son sus teorías implícitas, es decir, "por qué actúa y planifica de determinada manera". 
Cuando el docente pueda explicitar eso, se dará cuenta de los errores que está cometiendo y eso lo hace a través de un proceso de indagación; entonces la investigación se convierte en su práctica y no en la del otro, como hacen algunos postgrados, investigar para el participante; es decir, el docente también debe investigar, no sólo para hacer su trabajo de ascenso, sino sobre su práctica en el postgrado.

Se observa coincidencia con lo planteado por Marval y Acosta (2001), las autoras enfatizan que los postgrados para sobrevivir a las exigencias del modelo hegemónico de mercado, deben responder a las presiones exógenas del momento. Para consolidarse deben establecer estrategias investigativas que les permitan detectar las necesidades de la comunidad a la cual debe servir; no solo las del momento sino también mantener un conocimiento permanente de las que vayan surgiendo en el devenir del tiempo para poder satisfacerlas. Es importante un equilibrio entre las exigencias verdaderamente esenciales de la sociedad y las respuestas que puedan dar estas instituciones educativas

Hallazgo 8: los estudios de postgrado, expresan las informantes, deben ser un espacio amplio, un espacio donde la reflexión, investigación, docencia, extensión y la productividad, existan y coincidan realmente las expectativas de la sociedad y lo que la universidad le ofrece a esa sociedad para poder atender sus necesidades.

Creencia coincidente con lo planteado por Marval y Acosta y (2004), para las autoras el postgrado debe sustentarse en una concepción educativa definida e innovadora, basada en la preparación de profesionales que se desempeñen en el ámbito científico y técnico; con una actitud participativa, crítica y creativa. Una concepción educativa basada en la búsqueda del desarrollo integral y autónomo del hombre, que partiendo de sus raíces históricas se dirija hacia la afirmación del pluralismo social con el fin de reafirmar su identidad cultural en la sociedad.

Hallazgo 9: para los informantes, la universidad no puede tener una concepción cerrada, todo lo contrario debe tener espacio para todas las concepciones; además hoy es necesario pensar en la 
educación individualizada, es decir, para cada individuo, pero ésta debe ser generada bajo una filosofía y una concepción amplia para formar a un ser crítico, pensante, socializado, con un entendimiento y sensibilidad social, para responder a esas expectativas y necesidades. Debe ser una ecuación para la diversidad de pensamientos.

De esa manera, el postgrado se constituye en una comunidad de académicos que conforman una institución al servicio de la sociedad, cuya función primordial es satisfacer sus necesidades de aprendizaje y conocimiento, bien sea formando profesionales de alto nivel educativo, haciendo investigación e innovando para contribuir al desarrollo socio-económico; difundiendo saberes a diferentes sectores del colectivo societal, todo ello con el fin de contribuir al desarrollo moral e integral de los ciudadanos.

Hallazgo 10: el análisis de la data nos permite afirmar que los estudios de postgrado deben ser interdisciplinarios y transdisciplinarios, se debe considerar el principio del respeto a la diversidad de ideas. Los postgrados no pueden estar enmarcados estrictamente dentro de una disciplina, ahora uno ve la posibilidad de pensar en un postgrado, ya sea especialización, maestría o doctorado bajo una óptica mucho más amplia y que, sin embargo, no pierda ese nivel de profundidad y de especialización.

Lo planteado anteriormente, permite reflexionar sobre los modelos curriculares con base en los cuales los postgrados sustentan los diseños curriculares de sus ofertas académicas. La nueva orientación académica apunta hacia la formación integral de los estudiantes mediante la conformación de un currículo flexible, transdisciplinario y transversal. Torres (2000), considera que este tipo de currículo permite que las actividades de aprendizaje se seleccionen tomando en cuenta tanto los requerimientos del programa como las características del estudiante. Cada alumno tiene la oportunidad de seleccionar su carga académica, de acuerdo con su interés y disponibilidad de tiempo.

Hallazgo 11: los informantes plantean que los estudios de postgrado deben mantener una red de relaciones interconectadas con la comunidad y el entorno, para que respondan a las necesidades de 
los participantes y del país. Además, deben tener puente de enlace con el pregrado, para satisfacer la necesidad de formación permanente y continua. El postgrado debe tener pertinencia social al dar respuesta a las necesidades locales, las regionales, las nacionales y también a las necesidades mundiales.

Al respecto Marval y Acosta (2004) afirman, es necesario que los postgrados avancen con apertura hacia otros ámbitos institucionales, en cuanto a la creación de conocimiento, es decir, compartir con el sector productivo la responsabilidad de profundizar en la formación de profesionales y especialistas en diversas áreas del conocimiento, lo cual implica de alguna manera validar curricularmente cierto tipo de actividades realizadas fuera del aula. Pocas fuentes parecerían tan promisorias para el postgrado en materia de pertinencia como la de ofrecer conocimientos y estar dispuesto a no imponer la oferta académica sin conocer los requerimientos de la demanda y tratar de satisfacerla. En el Gráfico 3, se muestra la representación visual de la concepción de los estudios de postgrado de la UNERMB, como resultado de los hallazgos que emergieron de la data.

\section{Implicaciones para investigaciones futuras}

Las investigadoras consideran pertinente abordar este estudio en otros postgrado de la región y del país, para ir construyendo una teoría más afinada sobre el fenómeno en estudio.

\section{Reflexiones de las investigadoras}

Incursionar en investigación cualitativa no constituyó un trauma para nosotras como investigadoras, en ningún momento, al contrario, queremos expresar que a pesar de ser novedosa desde todo punto de vista, era algo en lo cual siempre creímos, debido a que este enfoque investigativo permite expresar sentimientos reales en cualquier conjunto de prácticas, además de un alto grado de compromiso con los lectores.

Investigar, en la tradición cualitativa de la TF, implicó un desarrollo mayor de nuestros sentidos, aprendimos a ser tolerantes, 


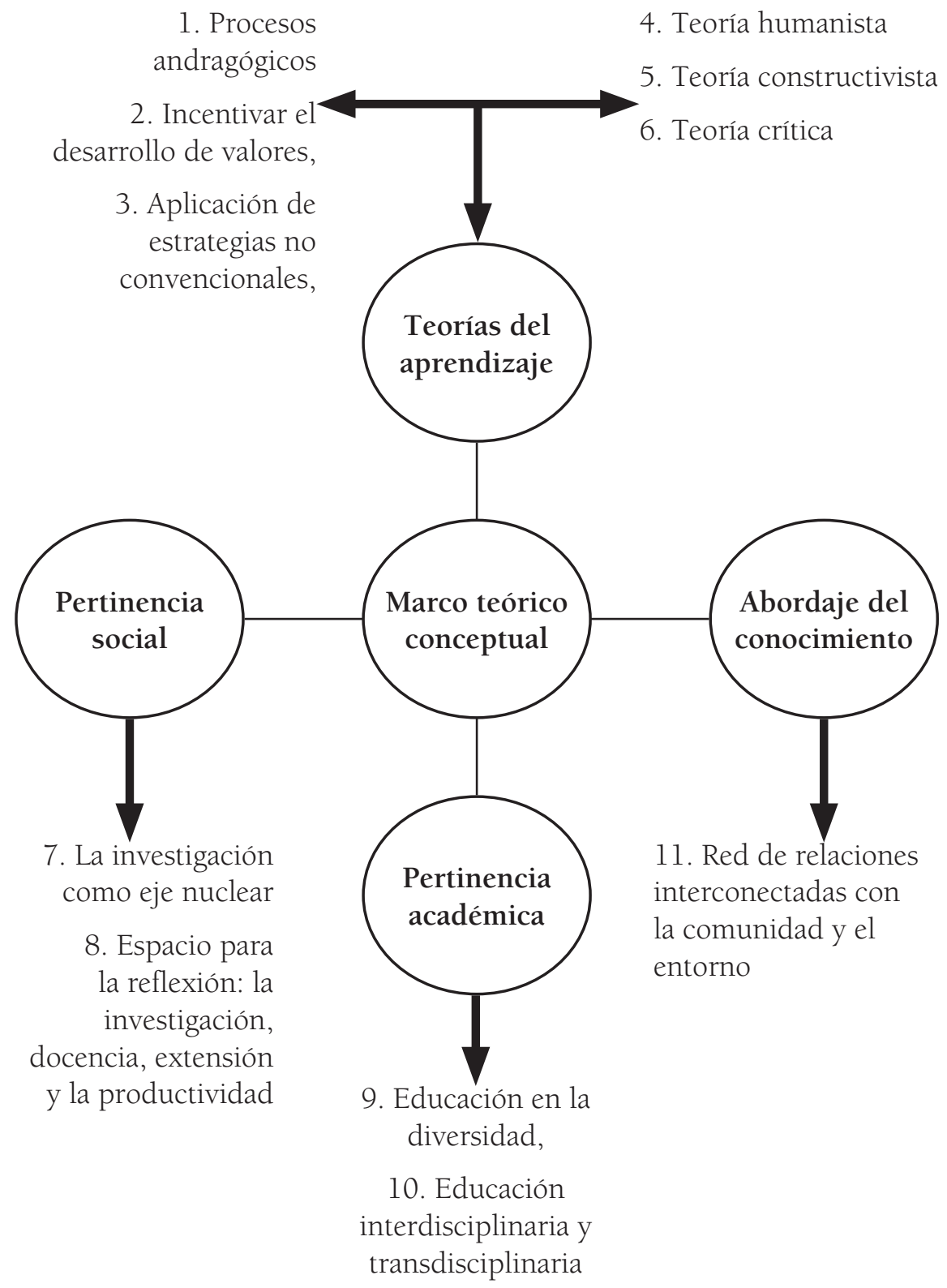

Gráfico 3. Representación visual de la concepción educativa de los estudios de Postgrado de la UNERMB. 
perseverantes y humildes para saber aceptar la diversidad de criterios y entender que cada quien es dueño de sus experiencias y de sus acciones. Para ello, tuvimos que despojarnos de la vanidad intelectual para hacerle saber a las informantes clave, que nuestra presencia allí era sólo para darle forma y significado a lo conocido plenamente por ellas, para encontrarle así significado a los datos.

Entendimos que mientras más sensible es el investigador a la pertinencia teórica de ciertos conceptos, más probable es que reconozca los indicadores de estos conceptos en los datos, tal como lo manifiestan Strauss y Corbin (2002 ${ }^{a}$, p. 224). La sensibilidad realmente fue creciendo durante el proceso de investigación y nos permitió decidir que conceptos buscar y de qué manera encontrar los indicadores para hacerlo.

Pensamos que la TF, es un método de investigador enriquecedor por su carácter dinámico y flexible, le da la oportunidad al investigador de ser creativo, reflexivo, organizado; pues puede volver a los datos tantas veces crea necesario y recodificarlos a la luz de los nuevos conocimientos que vayan surgiendo.

Como investigadoras mantuvimos una estrecha forma de cooperación e interacción basada en nuestras experiencias y conocimientos; pero también aprovechamos nuestras fortalezas y lo más importante, respetamos nuestras debilidades para llegar triunfantes a la meta. Fueron muchas horas de dedicación, observación, investigación, análisis e inquietudes, las cuales son inevitables, por mucha práctica, relajación o respiración, para citar algunas de las estrategias holísticas que practicamos.

Nos dimos cuenta que al investigar haciendo uso de la TF, se transita por un proceso metódico, riguroso, sistemático y lento, pero placentero porque la posibilidad de generar teoría despertó en nosotras curiosidad, fundada por el deseo y sensibilidad de ahondar en la naturaleza evolutiva y el desarrollo permanente de los acontecimientos, de allí la decisión por la TF.

La experiencia de compartir el relato de las informantes clave, sus vivencias y sueños por un postgrado mejor, sus reflexiones acerca de sus experiencias, su desempeño profesional, pero sobre todo su 
disposición y la dimensión humana que predican, fue gratificante, nos permitió recolectar un cúmulo de conocimientos y fortalecer las aspiraciones iníciales con mayor profundidad, pues su sencillez y disposición se vieron reflejadas para dar luz a este transitar.

\section{Referencias}

Creswell, J. (1998). Qualitative inquirí and research design. Choosing among five tradition. California: Sage.

Glasser, B. G. y Strauss, A. L. (1967). The discovery of grounded theory. Chicago: Aldine.

Marval, E. (2001). Pertinencia social de los estudios de postgrado: Una metodología de evaluación. Trabajo de ascenso. Universidad Nacional Experimental Rafael María Baralt. Cabimas.

Marval, E. y Acosta, I. (2001). Concepción y necesidades de estudios de Postgrado desde la perspectiva del Sistema Nacional de Educación Avanzada. (Caso: Postgrado de la Universidad Nacional Experimental Rafael María Baralt. UNERMB). Revista Agenda Académica. Vol. 8. No. 1: 51-61.

Marval, E. y Acosta, I. (2004). Transdisciplinariedad y transversalidad en los estudios de postgrado de la Universidad Nacional Experimental Rafael María Baralt. Revista Anales. Vol. 4. No. 2: 63-81.

Marval, E. y Valera, K. (2007). Perfil por competencias del facilitador de postgrado: un estudio cualitativo, según la teoría fundamentada. Revista Venezolana de Ciencias Sociales. Enero-junio, Vol. 11. No. 1: 60-77.

Maxwell, J. (1996). Qualitative research design an interactive approach. Thousand Oaks, California: Sage Publications.

Morles, V. (2007). La universidad latinoamericana actual: necesidad de replantear su misión. Repositorio Saber - ULA. Recuperado de http://blog.ucsar.com/wp-content/uploads/2007/11/ la-universidad-latinoamericana.pdf.

Román, E y Peña, M. (2006). Modelo de hecho educativo integrativo. Material mimeografiado. Línea de Investigación: Dimensión 
Humana de las Situaciones de Aprendizaje (Dihusa). Universidad Rafael María Baralt. Programa Posgrado. Cabimas

Rusque, A. M. (1999). De la diversidad a la unidad en la investigación cualitativa. Caracas: Ediciones Faces/UCV.

Sandín, M. (2003). Investigación cualitativa en educación. Fundamentos y tradiciones. España: Mc Graw-Hill.

Strauss, A. y Corbin, J. (2002a). Bases de la investigación cualitativa: Técnicas y procedimientos para desarrollar la teoría fundamentada. Medellín: Editorial Universidad de Antioquia.

Strauss, A. y Corbin, J. (2002b). Fundamentos de la investigación cualitativa: Técnicas y procedimientos para desarrollar teoría fundamentada. Thousand Oaks, California: Sage.

Taylor y Bogan (1990). Introducción a los métodos cualitativos de investigación. Argentina: Editorial Paidós.

Torres, J. (2000). Globalización e interdisciplinariedad: el currículum integrado. España: Ediciones Morata

Vera, G. (2003a). Estudio Piloto: La práctica educativa en orientación. (Un diseño de investigación cualitativa fundamentada). Revista Omnia, año 9, No. 2. Maracaibo: Universidad del Zulia.

Vera, G. (2003 b). Perspectiva en la formación de orientadores: Un estudio cualitativo según la teoría fundamentada. Trabajo de Ascenso no publicado para optar a la categoría de Titular. Maracaibo: Universidad del Zulia. 